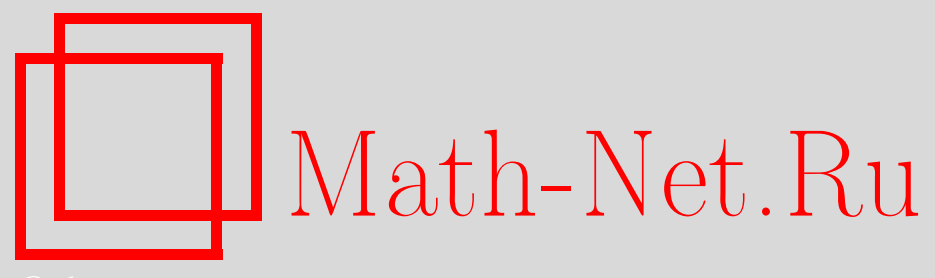

Е. Бакаев, Периметр и площадь на клеточках, Квант, 2019, номер 12, 19-22

DOI: https://doi.org/10.4213/kvant20191203

Использование Общероссийского математического портала Math-Net.Ru подразумевает, что вы прочитали и согласны с пользовательским соглашением http://www.mathnet.ru/rus/agreement

Параметры загрузки:

IP: 54.224 .187 .69

26 апреля 2023 г., 11:29:11

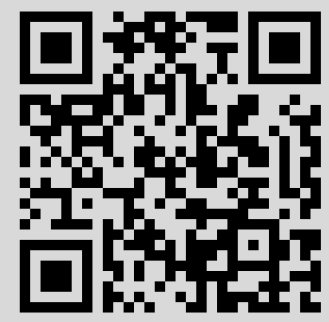




\section{Периметр и площадь на клеточках}

\section{E. БAKAEB}

$\mathrm{B}^{3}$ ЭТОЙ СТАТЬЕ РЕЧЬ ПОЙДЕТ О КЛЕточных фигурках - таких фигурках, которые составлены из клеточек листа клетчатой бумаги. Как связаны площадь и периметр такой фигурки? Среди всех фигурок одной и той же площади у каких периметр наибольший? Вот основные вопросы, с которыми мы разберемся.

Решать упражнения не обязательно для понимания дальнейшего материала, но они позволяют лучше разобраться с пройденным, так что рекомендуем уделить им внимание. Ответы и указания к ним даны в конце журнала. Решения задач даны сразу после условий; над ними тоже лучше сначала поразмышлять самостоятельно.

Условимся, что фигурки, о которых пойдет речь, являются связными. Это значит, что от любой клетки фигурки можно дойти до любой другой, переходя в соседние по стороне клетки. Например, множества клеток $A, B$ и $B$ на рисунке 1 - это фигурки, но $\overline{\text { и }} B$ вместе не образуют одну фигурку,

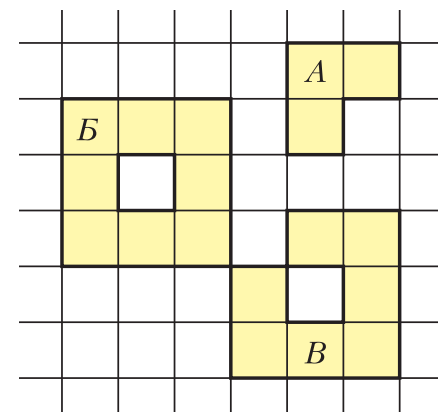

Pис. 1

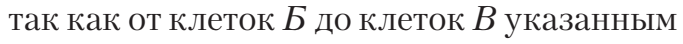
способом дойти нельзя.

Периметром фигурки называют длину ее границы. Например, у фигурки $A$ периметр 8 , а у $Б$ и $B-$ по 16 .

DOI: https://doi.org/10.4213/kvant20191203
Некоторые (но не все!) клеточные фигурки являются многоугольниками (напомним, что граница многоугольника должна быть замкнутой несамопересекающейся ломаной, иначе говоря, многоугольник - это фигурка «без дыр») . Например, фигурка $A$ является многоугольником, а фигурки $Б$ и $B-$ нет.

Введем некоторые новые понятия. В каждой клетке фигурки будем писать, сколько сторон этой клетки лежит внутри фигурки. Назовем это

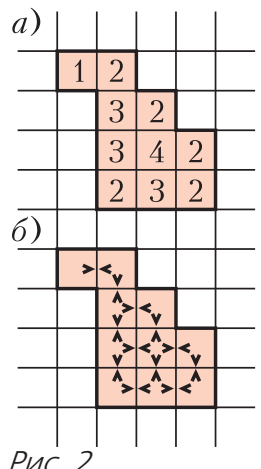
внутренним набором чисел фигурки. Пример показан на рисунке 2, $a$.

Упражнение 1. Существует ли фигурка с таким внутренним набором чисел:
a) $1,1,1,1,4$
б) $0,1,2,3,4$;
в) $1,1,1,1$;
г) $2,2,2,2,3,3,3,3,4$ ?

Задача 1. Докажите, что сумма чисел внутреннего набора фигурки всегда четна.

В сумме чисел внутреннего набора, будем обозначать ее $\sum_{\text {внутр }}$, каждая внутренняя перегородка между клетками посчитана по 2 раза (рис.2,б). Таким образом, $\sum_{\text {внутр }}=2 i$, где $i-$ количество внутренних перегородок.

Теперь в каждой клетке фигурки будем писать, сколько сторон этой клетки лежит на границе фигурки. На-

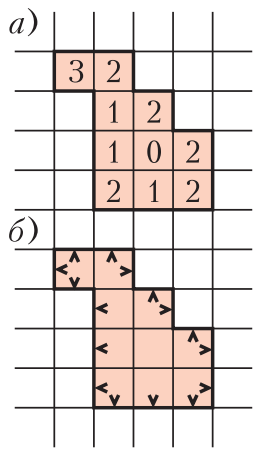

Pnc. 3 
зовем это внешним набором чисел фигурки. Пример приведен на рисунке 3,a.

Упражнение 2. Существует ли фигурка с таким внешним набором чисел:
a) $0,3,3,3,3$;
б) $0,2,3,3$;
в) $0,0,2,2,2,2,2,2,2,2,2,2,2,3,3,3,3,3,3$;
г) $1,1,1,1,1,1,1,1,1,1,1,1,1,1,1,1$ ?

Задача 2. Как по внешнему набору чисел найти периметр фигурки?

Во внешнем наборе каждый единичный отрезок периметра посчитан один раз (рис.3,б). Значит, сумма чисел внешнего набора равна периметру: $\sum_{\text {внешн }}=p$.

Теперь используем найденные свойства внутреннего и внешнего наборов для вывода полезной формулы.

Задача 3. Пусть s - это площадь фигур ки, $p$ - ее периметр, $a i$ - количество внутренних перегородок. Найдите и докажите формулу, связываюшую эти три величины.

Поймем, как связаны внешний и внутренний наборы. В одном случае мы для каждой клетки считали, сколько из ее сторон лежит внутри, а в другом - сколько на границе. Таким образом, в двух наборах вместе мы посчитали все 4 стороны каждой из $s$ клеток. Значит, $\sum_{\text {внутр }}+\sum_{\text {внешн }}=4 s$. Используя результаты предыдущих задач, получим:

$$
2 i+p=4 s .
$$

Упражнение 3. Найдите все фигурки, у которых периметр больше площади

а) в 5 раз; б) в 4 раза;

в) в 3 раза.

Из доказанной формулы следует такое свойство

Задача 4. Докажите, что периметр клетчатой фигурки всегда является четным числом.

Из формулы видно, что $p$ является разностью четных чисел $4 s$ и $2 i$, поэтому оно тоже четно.

В следующих упражнениях снова помогает та идея, что при суммировании периметров частей разрезы учитываются дважды.

\section{Упражнения}

4. Квадрат $5 \times 5$ разрезали на несколько многоугольников так, что суммарная длина разрезов равна 12. Чему может быть равен суммарный периметр всех многоугольников?

5. Доску $6 \times 6$ разрезали на прямоугольники $1 \times 2$. Какой может быть суммарная длина разрезов?

6. Квадрат $8 \times 8$ разрезали на тетраминошки (фигурки из четырех клеток). Суммарная длина разрезов оказалась равна 60. Сколько среди этих фигурок может быть квадратов?

Теперь обсудим следующую задачу.

Задача 5 (Н.Стрелкова). Квартира $3 \times 3$ cостоит из 9 квадратных комнат $1 \times 1$. Каждые две соседние комнаты соединены дверью. Какое наименьшее количество дверей должно быть открыто, чтобы кот, находящийся в одной из комнат, мог свободно гулять по всей квартире?

Территорией будем называть множество комнат, между которыми можно свободно передвигаться. Если от одной комнаты нельзя дойти до другой, то они отнесены к разным территориям. (В примере на рисунке 4 территория кота состоит

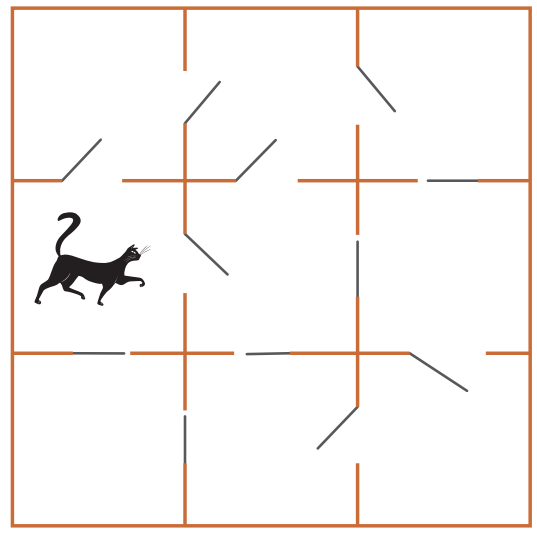

Рис. 4

из 5 комнат, а общее количество территорий равно 3.)

Первое решение (размер территории кота). Сначала закроем все двери и будем открывать их по порядку. Пусть этот порядок выбирает сам кот. Он может подойти к любой закрытой двери и мяукнуть, и тогда мы откроем ему эту дверь. Если она ведет в новую комнату, территория кота 
увеличится на одну комнату. Если же эта дверь ведет в комнату, до которой кот уже мог дойти иным путем, то территория не изменится. Получается, с каждой открытой дверью территория кота увеличивается не больше чем на 1. Сначала она равна 1, а в конце - 9. Значит, потребуется открыть хотя бы 8 дверей.

Задача решена. Подумайте, где именно используется, что мы предлагаем выбрать дверь коту, а не открываем ее сами.

\section{Второе решение (количество террито-} рий). Снова закроем все двери и будем открывать их по одной. Теперь будем считать общее количество территорий. Сначала каждая комната образует одну территорию, т.е. всего территорий 9. В конце все комнаты образуют одну территорию. Дверь может соединять либо две разные территории - тогда при ее открывании количество территорий уменьшится на 1, либо соединять территорию с самой собой - тогда количество территорий не уменьшится. Снова выходит, что хотя бы 8 дверей должны быть открыты.

В следующем упражнении помогает тот же прием.

Упражнение 7. В квадратном лабиринте размером $6 \times 6$ от любой клетки можно дойти до любой другой. Какое наибольшее количество внутренних перегородок может в нем быть?

Теперь применим этот подход к клетчатым фигуркам.

Задача 6. Какое наименьшее количество внутренних отрезков сетки может быть в фигурке с плошадью $s ?$

Рассмотрим произвольную фигурку площади $s$, покрасим ее всю в белый цвет. Выберем одну из клеток фигурки, покрасим ее в черный. Далее будем закрашивать по одной белой клетке в черный цвет так, чтобы новая черная клетка примыкала по стороне к уже закрашенной части. (Такую клетку всегда получится найти: если есть и белая клетка, и черная, то на пути от одной до другой встретится пара из двух соседних разноцветных клеток.) Каждый раз количество внутренних отрезков сетки в черной фигурке будет увеличиваться хотя бы на единицу. В итоге все клетки станут черными. Значит, внутренних отрезков исходной фигурки не меньше $s-1$.

Заметим, что алгоритм добавления клеток здесь ровно такой же, что и в задаче про кота.

Легко привести пример, в котором внутренних отрезков действительно столько: это, например, прямоугольник $1 \times s$.

Задача 7. Какой наибольший периметр может быть у фигурки с площадью $s ?$

Нами уже доказана формула $2 i+p=4 s$. Из нее видно, что при фиксированном $s$ чем больше $p$, тем меньше $i$. Значит, периметр наибольший тогда, когда количество внутренних отрезков наименьшее. Как мы уже выяснили, это достигается в случае $i=s-1$. Подставив это в формулу $2 i+p=4 s$, получим, что $p=2 s+2$. Итак, периметр фигурки с площадью $s$ не больше чем $2 s+2$. Пример подходит прежний.

Попробуйте применить ту же идею последовательного добавления клеток в следующих упражнениях.

\section{Упражнения}

8. Из фигурки, состоящей из 20 клеток, можно вырезать квадрат $3 \times 3$. Докажите, что ее периметр не больше 34 .

9. Известно, что некоторую клеточную фигурку можно разрезать на 5 квадратов $2 \times 2$. Какой наибольший периметр у нее может быть?

10. Нарисуйте по 10 разных фигурок, у каждой из которых периметр равен 14, а площадь равна а) 6; б) 7 .

в) Почему фигурки из пункта б) содержат квадрат $2 \times 2$, а фигурки из пункта а) - нет?

Разберем теперь задачу, где можно применить доказанную в задаче 7 оценку периметра.

Задача 8. Можно ли квадрат $5 \times 5$ разрезать на два клетчатых многоугольника, у каждого из которых периметр равен 28?

Предположим, что можно так разрезать. Дальше можно действовать по-разному.

Первое решение. Так как сумма площадей фигурок равна 25, то у одной из полученных фигурок площадь не больше 12. По предыдущей задаче, ее периметр не больше $2 \cdot 12+2=26$. Противоречие. 
Второе решение. Пусть длина разреза равна $v$, тогда суммарный периметр фигурок равен $20+2 v$, а по условию это $28 \cdot 2$. Из полученного равенства находим $v=18$. Так как частей две, то разрез один и не может разветвляться - он представляет собой одну цепочку отрезков, проходящую по узлам внутри квадрата $5 \times 5$. (Поясним это подробнее. Если узел находится на разрезе, то из него выходит ровно два отрезка разреза. Ведь один отрезок выходить не может, и 3 или 4 также не могут потому что фигур две и разрез должен разделять две разные фигуры. Значит, если пойти от границы по разрезу, то будем всегда идти по линии без развилок.)
Итак, разрез - это цепочка из 18 отрезков, значит, на ней лежит 17 узлов. Но внутри квадрата находится $4 \cdot 4=16$ узлов - на 1 меньше минимального необходимого количества. Противоречие.

Напоследок заметим, что в задачах 5 и 6 речь по сути идет о теореме из теории графов: в связном графе на п вершинах не менее $n-1$ ребер. Соответственно, эту теорему можно доказать аналогично задаче 5. Подробнее о графах говорить не будем: вместо этого порекомендуем статью П.Кожевникова и А.Шаповалова «Свяжитесь с графом» («Квант» №4 за 2014 г.), посвященную задачам, в которых скрыта упомянутая теорема.
(Начало см. нас. 13)

равняться внутреннему сопротивлению батарейки. Это означает, что нужно подключить к батарейке резистор сопротивлением

$$
R^{\prime}=\frac{R}{4}
$$

Максимальная мощность в этом случае будет равна

$$
P_{\max }=\frac{\delta^{2}}{R}=\frac{25}{16} I^{2} R .
$$

Ф2584. Молоко жирностью 3,2\% пролили на лист бумаги с напечатанным мелким тестом. Через слой молока толщиной 0,1 мм текст еше читается, а через более толстьй слой - нет. Оцените размеры капелек жира в таком молоке.

Поскольку размеры капелек жира требуется оценить, а не вычислить точно, то для простоты расчетов можно принять, что все капельки масла, взвешенные в воде, имеют такую же плотность, как и вода, а их форма не шарик, а кубик с ребром $D$. Обозначим концентрацию капелек жира через $n$, тогда из условия задачи, очевидно, следует, что

$$
n D^{3}=0,032 .
$$

Жир имеет отличающийся от воды коэффициент преломления, поэтому на стенках капелек свет частично отражается, а частично проходит сквозь границу раздела. Это рассеяние света молоком и приводит к эффекту «исчезновения» темных букв текста на фоне белого рассеянного света. Если на слой молока с очень малой толщиной $x$ порядка $D$ падает свет и площадь, на которую он падает, равна $S=D^{2}$, то доля света, прошедшего через воду и не попавшего ни на одну капельку жира, равна

$$
p=\left(1-x n D^{2}\right) \text {. }
$$

Пусть расстояние, пройденное светом в молоке, равно $L$, тогда соответствующая доля прошедшего света, не рассеянного капельками, составляет

$$
p^{L / x}=\left(1-x n D^{2}\right)^{L / x}=\exp \left(-L n D^{2}\right) .
$$

Согласно условию задачи при слое молока толщиной 0,1 мм текст уже не виден. А этот слой свет проходит дважды, т.е. $L=2 \cdot 0,1$ мм. «Текст не виден» означает, что контрастность видимой картины распределения света и темноты стала меньше, например, чем 10\%. Тогда

$$
\exp \left(-2 L n D^{2}\right)=0,1, \text { откуда } n D^{2} \approx 1 .
$$

Из двух соотношений для размеров капелек $D$ и концентрации $n$ получаем оценочное значение для размеров капелек жира: $D \approx 3 \cdot 10^{-6}$ м. 